\title{
Cortical Representation of Bimanual Movements
}

\author{
Uri Rokni, ${ }^{1}$ Orna Steinberg, ${ }^{3}$ Eilon Vaadia, ${ }^{2,3}$ and Haim Sompolinsky ${ }^{1,2}$ \\ ${ }^{1}$ Racah Institute of Physics and ${ }^{2}$ Center for Neural Computation, The Hebrew University, Jerusalem 91904, Israel, and ${ }^{3}$ Hadassah Medical School, \\ The Hebrew University, Jerusalem 91120, Israel
}

It is well established that the discharge of neurons in primate motor cortex is tuned to the movement direction of the contralateral arm. Interestingly, it has been found that these neurons exhibit a directional tuning to the ipsilateral arm as well and that the preferred directions to both arms tend to be similar. A recent study showed that motor cortex cells are also directionally selective to bimanual movements, but the relationship between the bimanual and unimanual representations remains unclear. To address this issue, we analyzed the responses of motor cortical neurons recorded from two macaque monkeys during unimanual and bimanual reaching movements. We decomposed the bimanual movement representation into contralateral and ipsilateral directionally tuned components. Our major finding is that the movement of the contralateral arm modifies the tuning of the cells to the ipsilateral arm such that: (1) the offset and modulation depth of the tuning are suppressed; and (2) the preferred directions are randomly shifted. Both these effects eliminate the correlation between the contralateral and ipsilateral representations during bimanual movements. We suggest that the modification of the ipsilateral arm representation is caused by the recruitment of local inhibition that conveys callosal inputs during bimanual movements. This hypothesis is supported by the analysis of a model of two motor cortical networks, coupled with sparse random interhemispheric projections that reproduce the main features observed in the data. Finally, we show that the modification of the ipsilateral arm representation reduces the interference between the movements of both arms.

Key words: motor cortex; directional tuning; movement representation; bimanual; network model; inhibition

Many motor tasks require fine coordination between the movements of both arms. Yet, very little is known about the neural mechanisms underlying bimanual coordination (for review, see Swinnen, 2002). It is well established that many motor cortical neurons are tuned to the direction of movement of the contralateral arm. These activities are often described by unimodal tuning curves with preferred directions (PDs) that are distributed uniformly (Georgopoulos et al., 1982). Additionally, it has been shown that MI neurons fire before and during movements of the ipsilateral arm as well (Lecas et al., 1986). In a recent study (Donchin et al., 1998; Steinberg et al., 2002), cells from the primary motor (MI), supplementary motor (SMA), and dorsal premotor (PMd) cortical arm areas of macaque monkeys were recorded while they operated two $x-y$ manipulanda, one with each hand, which moved cursors on a video screen. The monkeys were trained to perform unimanual reaching movements to one of eight targets and bimanual movements to these same targets, in which two arms moved either in parallel or in opposite directions. These experiments showed that many cells exhibit unimodal tuning curves in both types of unimanual movements and both types of bimanual movements (Fig. 1a,b). An important feature of the data is that the PDs of the unimanual contralateral

Received Sept. 4, 2003; revised Nov. 2, 2003; accepted Nov. 2, 2003.

This work was partially supported by a grant from the USA-Israel Binational Science Foundation and by a center of excellence grant $(8006 / 00)$ from the Israel Science Foundation. Data recordings were made by S. Cardoso De Oliveira, 0. Donchin, 0. Steinberg, and A. Gribova.

Correspondence should be addressed to Dr. Haim Sompolinsky, Racah Institute of Physics, The Hebrew University, Jerusalem 91904, Israel. E-mail: haim@fiz.huji.ac.il.

Copyright $\odot 2003$ Society for Neuroscience 0270-6474/03/2311577-10\$15.00/0 and ipsilateral tuning curves are highly correlated (Steinberg et al., 2002) (Fig. 2a).

These results raise the question of the relationship between the bimanual representation and the two unimanual representations. If the underlying neural mechanism is linear, the bimanual-related neuronal activities should be the superposition of the activities taking place during the two constituent unimanual movements. However, a previous analysis of these data invalidates this linear hypothesis (Donchin et al., 2002). Figure $1 b$ illustrates the lack of fit of the bimanual response of a sample cell (solid lines) to the linear hypothesis prediction (dashed lines), namely that the parallel tuning curve is predicted by the linear hypothesis to be the sum of the unimanual tuning curves and that the opposite tuning curve is predicted to be the sum of the unimanual contralateral tuning curve with the inverted ipsilateral tuning curve. Motivated by the failure of the linear hypothesis, we further studied the nature of the bimanual responses of motor cortical cells from the recordings of the above-mentioned experiments. Here, we show that the movement of the contralateral arm modifies the representation of the ipsilateral arm. Next, we propose a mechanism of callosal inhibition to explain this effect and present a network model that supports this hypothesis.

\section{Materials and Methods \\ Data analysis}

The data. Our data include recordings from cells in MI, SMA, and caudal PMd cortical areas of two macaque monkeys during performance of a unimanual and bimanual center-out task (Donchin et al., 2002, their penetration maps). The monkeys performed movements of each arm or both arms in parallel or opposite directions, in which the different con- 
a

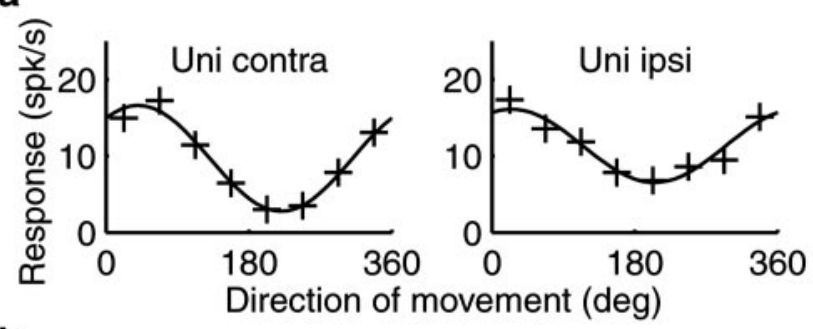

b

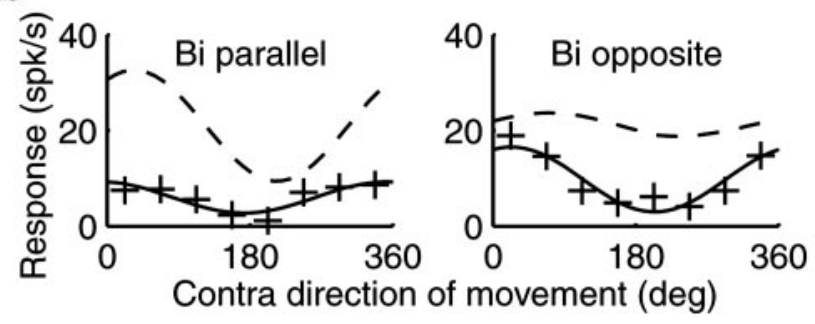

C
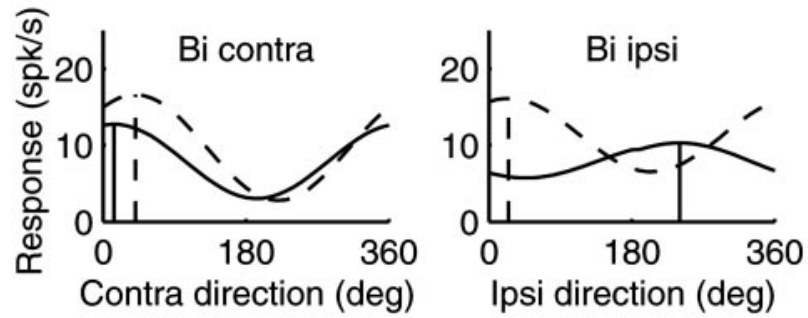

Figure 1. Example of directional tuning of one cell from Ml. $a$, Unimanual contralateral (left) and ipsilateral (right) tuning curves $(+)$ and cosine fits (solid lines). b, Bimanual parallel (left) and opposite (right) tuning curves $(+$ ), cosine fits (solid lines), and the bimanual cosine tuning expected according to the linear hypothesis (dashed line). c, We obtain the bimanual contralateral (left) and ipsilateral (right) components defined in Equation 9 (solid lines) with PDs $\theta_{b i}^{C}$ and $\theta_{b i}^{\prime}$, respectively (solid vertical lines), by summing and subtracting the parallel and opposite tuning curves in $b$, respectively. The dashed lines represent the linear hypothesis prediction.

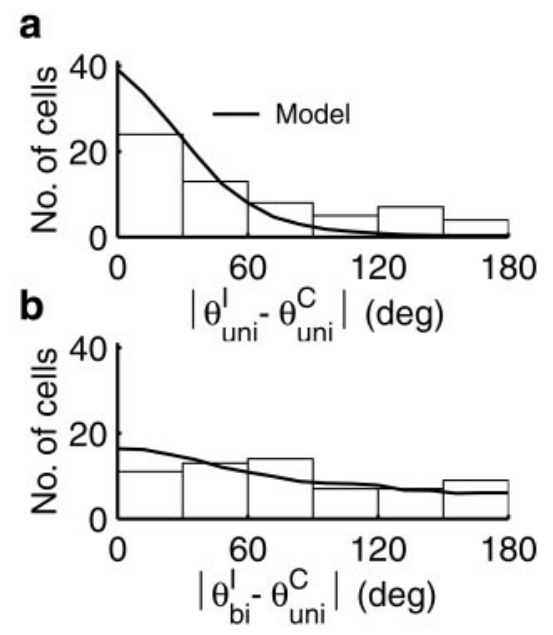

Figure 2. The correlation between the PDs of the contralateral and ipsilateral representations. $a$, Histogram of differences between the unimanual contralateral PD $\theta_{\text {uni }}^{c}$ and the unimanual ipsilateral PD $\theta_{\text {uni }}^{\prime}$ (bars) and the network model prediction (solid line). $b$, Histogram of differences between the unimanual contralateral PD $\theta_{u n i}^{C}$ and the bimanual ipsilateral PD $\theta_{b i}^{\prime}$ (bars) and the network model prediction (solid line).

ditions were shuffled pseudo-randomly (Steinberg et al., 2002). In our analysis, we characterized the response of a cell in a given trial by the mean rate, averaged over an epoch of -100 to $400 \mathrm{msec}$ relative to movement onset, minus the baseline rate before this epoch. To obtain a good estimate of the tuning parameters defined in Equations 7 and 9, we preselected cells that had tuning curves that are well described by a cosine. We first computed the $r^{2}$ fit to a cosine for each of the four tuning curves of each cell. Next, we averaged the four $r^{2}$ values of each cell and selected 61 cells that had a mean $r^{2}$ value that exceeded 0.7 . We note that our main results are not sensitive to the value of this threshold, or to our particular choice of selection criterion. The selected cells include 33 cells from the MI area (two monkeys), 18 cells from the SMA (one monkey), and 10 cells from the caudal PMd area (one monkey). The results we present are found in all areas and for both monkeys, in line with additional comparisons in previous work (Donchin et al., 2002). In the present analysis, all cells were pooled.

Statistical tests. We performed several tests to detect deviations from the null hypothesis of linear bimanual responses (see Eq. 8). According to this hypothesis, the mean of the bimanual offsets is equal to the mean of the sum of unimanual offsets (see Fig. 4). To test deviations from this hypothesis, we assumed that both these averages are normally distributed with the same mean and different variances that we estimated from the measured trial-to-trial variability. We applied a similar test to the difference in the variances of the bimanual and sum of unimanual offsets. According to the null hypothesis, the histograms in Figure $5 a$ should be concentrated at zero, with a certain spread caused by noise attributable to trial-to-trial variability. To test the null hypothesis, we generated an artificial histogram by taking the bimanual responses as the sum of the unimanual responses plus noise. The added noise was Gaussian with a variance assessed according to the measured trial-totrial variability. The probability that the artificial and experimental histograms were generated from the same distribution was assessed by a one-tailed Mann-Whitney $U$ test. This probability was averaged over 100 noise realizations. According to the null hypothesis, the points in Figure $5 b$ are equally likely to be above and below the linear prediction (solid line). Our H1 hypothesis is that points should tend to be below the line, and the statistic we used is the number of points below the line.

\section{Single-cell model}

The inputs that we assume for the single-cell model during unimanual contralateral and ipsilateral arm movements, respectively, are:

$$
\begin{gathered}
u^{C}\left(\varphi_{C}\right)=\beta^{C}+\alpha^{C} \cos \left(\varphi_{C}-\psi^{C}\right) \\
u^{I}\left(\varphi_{I}\right)=\beta^{I}+\alpha^{I} \cos \left(\varphi_{I}-\psi^{I}\right) .
\end{gathered}
$$

Here, $\varphi_{C, I}$ are the direction of movement of the hands. The tuning parameters of the inputs are the PDs $\psi^{C, I}$, the amplitudes $\alpha^{C, I}$ and the offsets $\beta^{C, I}$. During unimanual movements, the cell receives the input corresponding to the moved arm alone, whereas during bimanual movements the cell receives the sum of both inputs. The output of our model cell $r$ is related to its total input $u$ by the nonlinear relation:

$$
r=\frac{u}{1+u}
$$

where $r$ is normalized by the maximal firing rate, and $u$ is normalized by the input level that causes the neuron to fire at half its maximal rate. The method of extracting the tuning parameters of the output is identical to the method applied to the experimental data. The computation presented in Figure $7 a$ includes 61 such cells with inputs in which tuning amplitudes $\alpha^{C, I}$ and offsets $\beta^{C, I}$ were randomly chosen between 0 and 1 and in which PDs $\psi^{C, I}$ were random with a preference for similar PDs for the two hands (as observed in the experimental responses).

\section{Network model}

Network equations. Our network model includes two coupled cortical networks with $\mathrm{N}$ excitatory neurons and $\mathrm{N}$ inhibitory neurons each (see Fig. $8 e$ ). We use equations of a semi-linear rate model to describe the 
Table 1. Parameter values of network model

\begin{tabular}{|c|c|c|c|c|c|c|c|c|c|c|c|}
\hline$N$ & $s$ & $h$ & $\alpha$ & $\beta$ & $T$ & $J$ & $K$ & $\gamma$ & $\lambda_{\min }$ & $\lambda_{\max }$ & $K_{\text {intra }}$ \\
\hline 2000 & 2.1 & 51.6 & 9.6 & 12 & 58.8 & 1 & 7 & 7 & 0 & 0.28 & 3 \\
\hline
\end{tabular}

Units for $s, h, \alpha, \beta$, and $T$ are in spikes per second, whereas the other parameters are nondimensional.

neuronal activities as a function of the instructed directions of movement for the left and right hands, $\varphi_{L}$ and $\varphi_{R}$, respectively:

$$
\begin{gathered}
\tau \frac{d r_{E, i}^{R} d t}{d t}=-r_{E, i}^{R}+\left[s+g_{i}^{R}\left(u_{i}^{R}\left(\varphi_{L}\right)-J r_{I, i}^{R}+\sum_{j=1}^{N} \lambda_{E, i j}^{R L} C_{i j}^{R L} r_{E, j}^{L}\right)\right]_{+} \\
\tau \frac{d r_{I, i}^{R}}{d t}=-r_{I, i}^{R}+\left[h+\sum_{j=1}^{N} \lambda_{I, i j}^{R L} C_{i j}^{R L} r_{E, j}^{L}-T\right]_{+} \\
\tau \frac{d r_{E, i}^{L}}{d t}=-r_{E, i}^{L}+\left[s+g_{i}^{L}\left(u_{i}^{L}\left(\varphi_{R}\right)-J r_{l, i}^{L}+\sum_{j=1}^{N} \lambda_{E, i j}^{L R} C_{i j}^{L R} r_{E, j}^{R}\right)\right]_{+} \\
\tau \frac{d r_{I, i}^{L}}{d t}=-r_{I, i}^{L}+\left[h+\sum_{j=1}^{N} \lambda_{I, i j}^{L R} C_{i j}^{L R} r_{E, j}^{R}-T\right]_{+},
\end{gathered}
$$

where $[x]_{+}$equals $x$ for positive $x$ and zero for negative $x$. The values of the model parameters are shown in Table 1. The superscripts $\mathrm{R}$ and $\mathrm{L}$ correspond to the right and left sides, and the subscripts E and I correspond to the excitatory and inhibitory populations. $\tau$ is the neuronal time constant, which is typically on the order of a few milliseconds, and $g_{i}^{R, L}$ are the gains of the excitatory cells, distributed uniformly between 0 and 1. The randomness of the gains is important for generating heterogeneous tuning amplitudes and offsets, as observed in the data. We assume that before the movement the sole input to the network is the baseline input of the excitatory cells $s$. When one arm is moved alone, its corresponding instruction input $u_{i}^{R}\left(\varphi_{L}\right)$ or $\left.u_{i}^{L}\right)\left(\varphi_{R}\right)$ is also present. When both arms are moved, both instruction inputs are present, and the control signal to the inhibitory cells $h$ is turned on. Because we are only interested in the mean firing rate during the movement, we assumed for simplicity that the inputs are constant throughout the movement. The matrices $C^{R L}$ and $C^{L R}$ describe the callosal connectivity, having elements with a value of 1 for connected pairs and 0 otherwise, and the matrices $\lambda^{R L}$ and $\lambda^{L R}$ are the random connection strengths. $J$ is the gain of the inhibitory interneurons, and $T$ is their threshold.

Instruction inputs. The instruction inputs are cosine tuned to the planned direction of movement:

$$
\begin{aligned}
& u_{i}^{R}\left(\varphi_{L}\right)=\beta+\alpha \cos \left(\varphi_{L}-\psi_{i}^{L}\right) \\
& u_{i}^{L}\left(\varphi_{R}\right)=\beta+\alpha \cos \left(\varphi_{R}-\psi_{i}^{R}\right) .
\end{aligned}
$$

Here, $\psi_{i}^{R, L}$ are the isotropically distributed PDs, $\alpha$ is the modulation amplitude, and $\beta$ is the offset of the instruction inputs.

Connectivity. We assume that the probability of having a callosal connection between a presynaptic neuron $j$ in the left hemisphere and a postsynaptic neuron $\mathrm{i}$ in the right hemisphere depends on the difference in the PDs of their instruction inputs:

$$
P\left(C_{i j}^{R L}=1\right)=\frac{1}{N}\left[K+\gamma \cos \left(\psi_{i}^{L}-\psi_{j}^{R}\right)\right],
$$

and similarly for $C_{i j}^{L R}$. Here, $K$ is the typical number of callosal afferents impinging on a cell, and $\gamma$ characterizes the degree of preference for connecting pairs with similar input PDs. The random connection strength matrix elements $\lambda_{i j}^{L R}$ and $\lambda_{i j}^{R L}$ are distributed uniformly between $\lambda_{\min }$ and $\lambda_{\max }$. In the simulations shown in Figure $9 d$, we added an internal connectivity term to the equations of the excitatory populations (first and third equations in Eq. 3). These terms included a random connectivity matrix $W_{i j}$, where $W_{i j}=w$ with probability $K_{\text {intra }} / N$ and $W_{i j}=0$ otherwise.
Directional tuning of the outputs. To extract the tuning parameters of the model neurons, we simulated the network equations (Eq. 3) for the following conditions: (1) premovement; (2) right arm movement; (3) left arm movement; (4) parallel bimanual movement; and (5) opposite bimanual movement. Because the network converges quickly to its steady state, on the order of neuronal time constant $\tau$, we assumed that the neuronal firing rates at the steady state correspond to the experimentally measured mean rates. For each of the four movement conditions, we computed the rates for eight different directions and subtracted the baseline rates computed for the premovement condition. Subsequently, we extracted the offsets, modulation amplitudes, and PDs by the same method that we used for the experimental data.

\section{Results}

\section{Bimanual and unimanual directional tuning functions}

We analyzed the relationship between the directional tuning of the bimanual and the unimanual responses of 61 well-tuned cells $\left(r^{2}>0.7\right.$; see Materials and Methods) from MI, SMA, and PMd. The directional responses of motor cortical cells during unimanual movements are often well approximated by cosine tuning curves:

$$
r(\varphi)=a \cos (\varphi-\theta)+b .
$$

$r(\varphi)$ is the modulation of the firing rate of a cell, minus the baseline rate before movement, as a function of the hand direction of movement. $a$ is the modulation amplitude, $\theta$ is the $\mathrm{PD}$, and $b$ is the offset. We describe the responses of a cell to the unimanual contralateral and ipsilateral movements by the following cosine tuning functions:

$$
\begin{aligned}
& r_{u n i}^{C}\left(\varphi_{C}\right)=a_{u n i}^{C} \cos \left(\varphi_{C}-\theta_{u n i}^{C}\right)+b_{u n i}^{C} \\
& r_{u n i}^{I}\left(\varphi_{I}\right)=a_{u n i}^{I} \cos \left(\varphi_{I}-\theta_{u n i}^{I}\right)+b_{u n i}^{I} .
\end{aligned}
$$

Here, the superscripts $C$ and $I$ denote contralateral and ipsilateral, respectively, and indicate that a cell may have different directional tuning parameters for each arm. The subscript uni distinguishes the tuning to unimanual movements from the tuning to bimanual movements.

A description of the response of cells to bimanual movements requires a tuning function of both directions of movement $\varphi_{C}$ and $\varphi_{I}$. If the linear hypothesis held, the bimanual tuning function would be the sum of the two unimanual tuning functions in Equation 7:

$$
r_{b i}\left(\varphi_{C}, \varphi_{I}\right)=a_{u n i}^{C} \cos \left(\varphi_{C}-\theta_{\text {uni }}^{\mathrm{C}}\right)+a_{\text {uni }}^{\mathrm{I}} \cos \left(\varphi_{\mathrm{I}}-\theta_{\text {uni }}^{\mathrm{I}}\right)+\mathrm{b}_{\text {uni }}^{\mathrm{C}}+\mathrm{b}_{\text {uni }}^{\mathrm{I}} \cdot
$$

However, because the linear hypothesis does not hold, we propose an alternative bimanual tuning function:

$$
r_{b i}\left(\varphi_{C}, \varphi_{I}\right)=a_{b i}^{C} \cos \left(\varphi_{\mathrm{C}}-\theta_{\mathrm{bi}}^{\mathrm{C}}\right)+\mathrm{a}_{\mathrm{bi}}^{\mathrm{I}} \cos \left(\varphi_{\mathrm{I}}-\theta_{\mathrm{bi}}^{\mathrm{I}}\right)+\mathrm{b}_{\mathrm{bi}} .
$$

Here, the bimanual tuning function is assumed to be the sum of an offset and two components, each cosine tuned to the direction of movement of one hand. In contrast to the linear hypothesis (Eq. 8), the amplitudes and PDs of these components are not necessarily identical to the amplitudes and PDs of the corre- 
sponding unimanual tuning curves, and the offset of the bimanual tuning curve is not necessarily equal to the sum of the unimanual offsets (as indicated by the subscript bi). The bimanual contralateral and ipsilateral components in Equation 9 describe the ways in which the bimanual response varies with the direction of movement of one hand when the direction of movement of the other hand is kept constant. Although our data are limited to parallel $\left(\varphi_{I}=\varphi_{C}\right)$ and opposite $\left(\varphi_{I}=\varphi_{C}+180^{\circ}\right)$ movements, we can extract the contralateral (ipsilateral) component of the bimanual response from the data by summing (subtracting) the parallel and opposite tuning curves. The solid lines in Figure $1 c$ depict the bimanual contralateral and ipsilateral tuning components of a sample cell as extracted from its parallel and opposite tuning curves in Figure $1 b$.

The experimental data support the form of the bimanual tuning that is assumed in Equation 9 in two ways. First, the tuning to parallel and opposite movements is predicted to have a cosine shape, as shown experimentally in a previous study (Steinberg et al., 2002). Second, the offsets of the parallel and opposite tuning curves of each cell should be equal. This prediction is confirmed by our data (Fig. 3a). Equation 9 serves to predict the response of a cell to bimanual movements in general directions different from the measured parallel and opposite tuning curves. For purposes of illustration, consider the tuning curve to bimanual movements in orthogonal directions when the parallel and opposite tuning curves are of equal modulation amplitude. We predict that the orthogonal PD is half way between the parallel and opposite PDs (Fig. 3b). If these PDs are either identical or opposite, the amplitude of the orthogonal tuning curve is predicted to be the same as that of the parallel and opposite tuning curves. In contrast, cells for which the parallel and opposite PDs are nearly $90^{\circ}$ apart are predicted to have substantially reduced tuning amplitudes in the orthogonal movement configuration.

\section{Nonlinearity of the bimanual representation}

We characterize the deviations from the linear hypothesis in the bimanual cortical representation by comparing the bimanual tuning parameters (Eq. 9) to the unimanual parameters of the same cells (Eq. 7). We refer to such deviations as nonlinearities of the bimanual movement representation. The linear hypothesis predicts that the bimanual offset $b_{b i}$ is equal to the sum of the unimanual offsets $b_{u n i}^{C}+b_{u n i}^{I}$. Figure $1 b$ demonstrates the failure of the linear prediction for one cell, for which the offsets of the parallel and opposite tuning curves (solid lines) are substantially smaller than the offset of the tuning curve predicted by the linear hypothesis (dashed lines). Moreover, according to the linear hypothesis, the bimanual PDs $\theta_{b i}^{C}, \theta_{b i}^{I}$ and amplitudes $a_{b i}^{C}, a_{b i}^{I}$ should be equal to the corresponding unimanual PDs $\theta_{u n i}^{C}, \theta_{u n i}^{I}$ and amplitudes $a_{\text {uni }}^{C}, a_{\text {uni }}^{I}$. In Figure $1 c$, we demonstrate the failure of this prediction by comparing the contra and ipsi unimanual tuning curves (dashed lines) with the corresponding bimanual components (solid lines), which we extracted from the bimanual tuning curves in Figure $1 b$. In this example, the nonlinearity is evident in the shift of the bimanual PD $\theta_{b i}{ }^{I}$ relative to the corresponding unimanual PD $\theta_{u n i}{ }^{I}$. We interpret this nonlinearity as a change in the representation of the movement of the ipsilateral arm when the contralateral arm also moves. As we show below, these types a

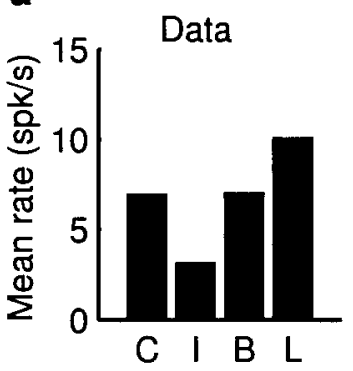

C
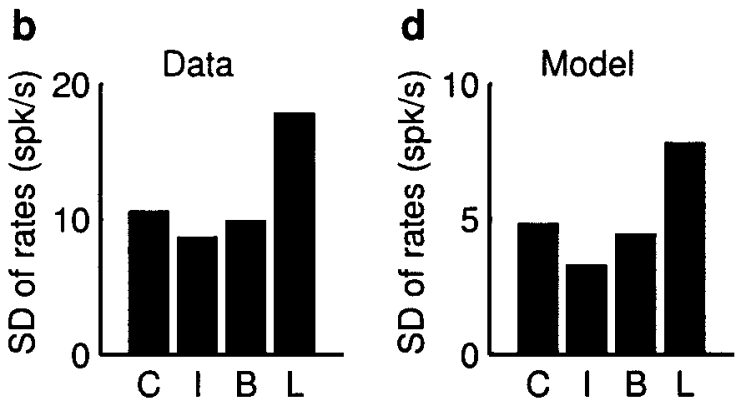

Figure 4. The bimanual offsets are smaller than the linear prediction. $a$, Means of the unimanual contra offsets $(C)$, unimanual ipsilateral offsets (I), bimanual offsets (B), and sum of unimanual offsets (L), over the population of cells. $b$, Standard deviations of the same sets of offsets as in $a$. $c, d$, The predicted means and SDs of the offsets according to the network model.

of nonlinearities in the offsets and modulations of the tuning curves are observed across the population of cells.

Figure 4, $a$ and $b$, depict the means and SDs across the population of cells of $b_{u n i}^{C}, b_{u n i}^{I}, b_{b i}$, and the linear prediction $b_{u n i}^{C}+b_{u n i}^{I}$, denoted C, I, B, and L, respectively. As shown, the mean and SD of the bimanual offsets are substantially smaller than the mean and SD of the linear prediction $(p<0.1 \%)$. Additionally, the bimanual and contralateral offsets are similar, as shown by their similar means and SDs and by their high correlation coefficient (0.92). This suggests that the suppression of the bimanual offsets is an outcome of a suppression of the ipsilateral representation rather than the contralateral representation.

The analysis of the directional modulation of the cell activities indeed shows that the nonlinearity is mainly attributable to the ipsilateral representation. The histograms in Figure $5 a$ reveal significant shifts of the bimanual PDs $\theta_{b i}^{C}$ and $\theta_{b i}^{I}$ relative to the corresponding unimanual PDs $\theta_{u n i}^{C}$ and $\theta_{u n i}^{I}(p<0.1 \%)$, whereas 

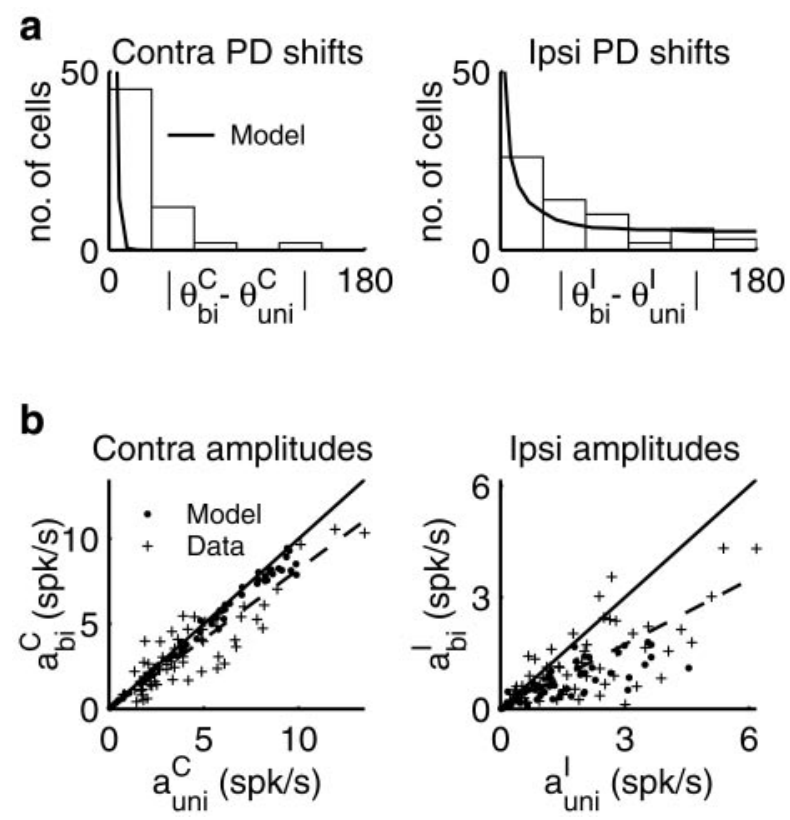

Figure 5. The directional tuning to the movement of one arm is modified when the other arm is moved as well. $a$, Histogram of the differences between the unimanual PDs and the bimanual PDs (bars), for both contralateral (left) and ipsilateral (right). The solid line depicts the distributions predicted by the network model. $b$, Scatter plot of the unimanual amplitudes of the cells versus the corresponding bimanual amplitudes (crosses), linear regression of data points (dashed line), and the linear hypothesis prediction (solid line), for both contralateral (left) and ipsilateral (right). The dots depict a sample of 61 cells from the network model.

the linear hypothesis predicts that these histograms should be concentrated near zero. However, the shifts in the ipsilateral PDs are much larger than the contralateral ones. The most striking aspect of the shift in the ipsilateral PDs is the fact that although the unimanual ipsilateral PDs $\theta_{u n i}^{I}$ are highly correlated with the unimanual contralateral PDs $\theta_{u n i}^{C}$, the bimanual ipsilateral PDs $\theta_{b i}^{I}$ are only weakly correlated with $\theta_{u n i}^{C}$ (compare histograms in Fig. 2). Note that the histogram of the differences between $\theta_{b i}^{I}$ and $\theta_{b i}^{C}$ (data not shown) is very similar to the histogram in Figure $2 b$, because of the high similarity of $\theta_{b i}^{C}$ and $\theta_{u n i}^{C}$. This means that the modification of the representation of the ipsilateral arm is such that it loses its correlation with the representation of the contralateral arm. Note that the histogram of the differences between $\theta_{b i}^{I}$ and $\theta_{b i}^{C}$ (data not shown) is very similar to the histogram in Figure $2 b$, because of the high similarity of $\theta_{b i}^{C}$ and $\theta_{u n i}^{C}$. In Figure $5 b$, we plot the bimanual modulation amplitudes versus the unimanual modulation amplitudes for all cells (crosses). The large spread, especially in the ipsilateral amplitudes, contradicts the linear hypothesis prediction (solid line). Additionally, for both the contralateral and the ipsilateral amplitudes, the slope of the linear regression (dashed line) is below the linear hypothesis prediction, showing a significant trend toward reduction of the bimanual amplitudes $(p<0.1 \%)$, similar to the suppressive effect seen in the offsets. In summary, the representation of the ipsilateral arm during the bimanual movements in our experiments is suppressed in its modulation amplitude and shifted in its PD, such that it loses its correlation with the contralateral arm representation. In contrast, the directional modulation of the contralateral representation is less suppressed and only slightly shifted in its PD. Additionally, the bimanual offsets are similar to the contralateral offsets and substantially smaller than the sum of the unimanual offsets.

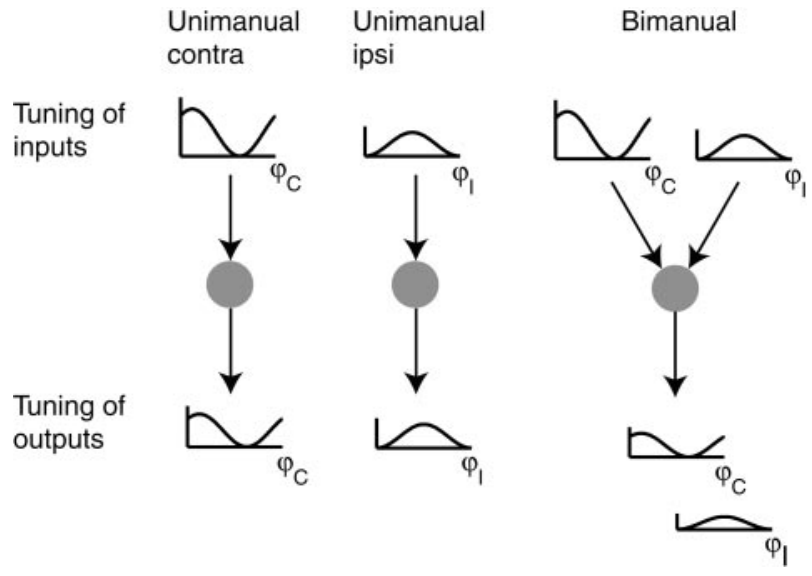

Figure 6. The single-cell mechanism. The input during a bimanual movement is the sum of the inputs during the two constituent unimanual movements. The nonlinearity of the bimanual response arises from output nonlinearity and causes a negligible PD shift.

\section{Failure of the single-cell model}

What underlying neuronal nonlinearity is responsible for the modification of the ipsilateral representation during bimanual movements? One class of possible mechanisms is the nonlinear response properties of single cells. We examined this idea by modeling a single motor cortical cell that receives two inputs from higher visuomotor areas (e.g. the parietal cortex and PMd) that are cosine tuned to the desired movement direction of the two hands, respectively. We assume that during a unimanual movement, only the input corresponding to the moved hand is present (Fig. 6) (see mathematical details in the Materials and Methods). The nonlinearity of the bimanual response is assumed to arise from the output saturating nonlinearity of the cell. The contralateral and ipsilateral components of the bimanual response, described in Equation 9, of the model neuron are extracted by the same method as in the data, and their tuning parameters depend on the tuning parameters of the inputs. We computed the tuning properties of 61 model cells with different input tuning parameters. Figure $7 a$ depicts the resulting ipsilateral PD shifts versus the difference between the two unimanual PDs $\left|\theta_{\text {uni }}^{I}-\theta_{\text {uni }}^{C}\right|$. This scatter plot reveals two major shortcomings of this model. First, in contrast to the large ipsilateral PD shifts observed in the data, the model predicts very small ipsilateral PD shifts (note the difference in the $y$-axis in Fig. 7a,b). Although output nonlinearity may distort a tuning function, the effect on the PD is usually small. A second shortcoming of the model is that these shifts are predicted to be highly correlated with the difference between the two unimanual PDs $\left|\theta_{\text {uni }}^{I}-\theta_{\text {uni }}^{C}\right|$, whereas the data show that the ipsilateral PD shift is largely independent of $\left|\theta_{u n i}^{I}-\theta_{u n i}^{C}\right|$ (Fig. 7, compare $\left.a, b\right)$. To understand the source of this correlation in the model, consider as an example the case in which the unimanual PDs for both hands are equal $\left(\theta_{u n i}^{I}=\theta_{u n i}^{C}\right)$. In this case, the single-cell model predicts that the parallel and opposite tuning curves should have a PD that is equal to $\theta_{u n i}^{C}$. It follows that the ipsilateral component of the bimanual response obtained by calculating the difference of the parallel and opposite tuning curves will also have a PD that is equal to $\theta_{u n i}^{C}$. Similar arguments show that there is no ipsilateral PD shift in the single-cell model when $\left|\theta_{u n i}^{I}-\theta_{u n i}^{C}\right|=90^{\circ}, 180^{\circ}$.

\section{Callosal inhibition mechanism}

Motivated by the failure of the single-cell model to produce large ipsilateral PD shifts, we suggest a network mechanism for the 
modification of the ipsilateral representation. The observation that the ipsilateral representation is modified substantially, whereas the contralateral is only slightly changed, suggests that the two representations are generated differently. Therefore, we propose that the inputs into a motor cortical cell, related to the contralateral arm movement, originate predominantly from the higher cortical areas in the same hemisphere, whereas the ipsilateral signals originate predominantly from the motor cortex in the opposite hemisphere. We refer to the former type of inputs as instruction inputs and the latter as callosal inputs. Hence, we assume that the callosal projections are excitatory. Another observation, namely the suppression of the ipsilateral representation, suggests that the callosal inputs are effectively suppressed during bimanual movements. We, therefore, pro-

pose the following biological mechanism for suppressing the callosal inputs: a callosal afferent that innervates a cortical cell also projects to the same cell via a local inhibitory interneuron (Fig. $8 a$ ). We assume that these interneurons are subthreshold during unimanual movements and are recruited during bimanual movements by an excitatory control signal from a higher brain area. Thus, the direct excitatory callosal projections generate the representation of the movement of the ipsilateral arm alone. In contrast, the indirect inhibitory callosal projections suppress the ipsilateral representation during bimanual movements. The role of the control signal is to activate these inhibitory callosal projections solely during bimanual movements. As we show below, this control signal can modulate the degree of suppression of the ipsilateral representation.

By way of demonstration, consider the gray cell that is shown in Figure $8 a$. This cell receives a callosal input from a single cell in the contralateral hemisphere. During a unimanual movement of the ipsilateral arm, the direct callosal excitatory pathway drives the cell and generates its tuning to the ipsilateral direction. Figure $8 b$ (Uni) shows the resulting tuning for a particular choice of connection weights. In contrast, during bimanual movements, the control signal recruits the inhibitory cell, thus adding an inhibitory input on top of the excitatory drive. Note that the excitatory and inhibitory inputs have the same PD, because they originate from the same source in the contralateral hemisphere. Therefore, the modulation amplitude and offset of the tuning curve of the cell are suppressed during bimanual movements (Fig. $8 b, \mathrm{Bi}$ ). The degree of the suppression depends on the relative strength of the excitatory and inhibitory pathways.

Interestingly, when several callosal inputs with different PDs converge onto the same cell, large ipsilateral PD shifts can occur. The basic idea is that the different tuned inputs are suppressed to different degrees during bimanual movements. As a result, the total callosal input into the cell has different PDs during unimanual and bimanual movements. For example, consider the excitatory cell shown in Figure $8 c$ that receives two callosal inputs with different PDs, through direct excitatory and indirect inhibitory pathways. During unimanual ipsilateral movements, the inhibitory interneuron is subthreshold, and the directional tuning of the cell is a weighted sum of the tuning curves of the two excitatory callosal inputs with a weight of 1 (Fig. $8 d$, Uni). However, during bimanual movements, the inhibition changes the b - Network model + Data

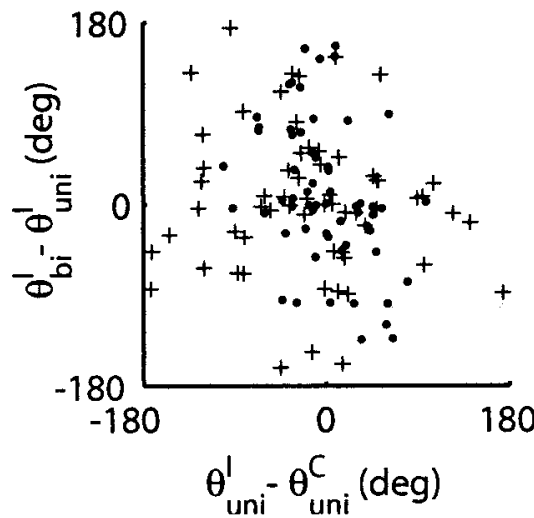
ipsilateral PD (Fig. 8d, Bi). Thus, heterogeneity in the tuning of the callosal inputs and their weights can generate large PD shifts. Note that similar effects can be generated by assuming that the sources of the callosal inputs at the contralateral hemisphere are suppressed.

\section{Network model of bimanual responses}

It remains to be demonstrated that the callosal inhibition mechanism quantitatively accounts for the tuning properties observed in the data. Additionally, in the above description, we ignored the reciprocal nature of the callosal interactions, namely that the sources of the callosal inputs themselves receive callosal inputs from the contralateral hemisphere. We, therefore, simulated a model of two interconnected networks representing the two hemispheric hand areas (Fig. 8e), each including an excitatory and inhibitory population of threshold-linear cells (see Materials and Methods for details). In accordance with the callosal inhibition hypothesis explained above, we assume that the excitatory neurons in each network receive cosine-tuned instruction inputs that convey the direction of movement of the contralateral arm, whereas the instruction for the ipsilateral arm is conveyed by the callosal afferents. A second ingredient of our hypothesis is that the source of nonlinearity is the recruitment of callosal inhibition solely during bimanual movements. We incorporate this mechanism into our model by assuming for simplicity that all callosal afferents that project onto an excitatory cell project to the same cell also via a local inhibitory interneuron (Fig. $8 a, c)$. We assume that these interneurons are recruited by a global excitatory control signal solely during bimanual movements. To compare the model with the experimental data, we computed the predicted distribution of the unimanual and bimanual tuning parameters over the population of excitatory cells.

In designing the architecture of the callosal connectivity in our network model, we aimed to account for two features observed in the unimanual tuning PDs (Fig. 2a): (1) the differences between the contralateral and ipsilateral PDs are heterogeneous; and (2) the two PDs are correlated. Models of cortical neuronal circuits often assume a high degree of convergence on each neuron (Ben-Yishai et al., 1995). This convergence tends to average out inhomogeneities in the strength of individual synapses or in the identity of the presynaptic sources of each neuron, leading to 
a

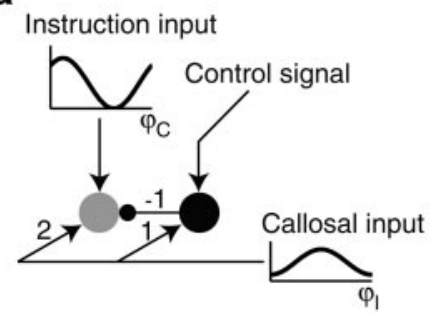

C

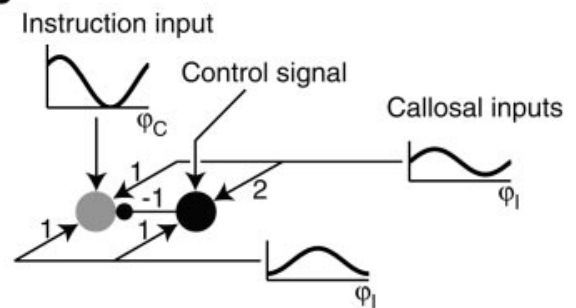

b

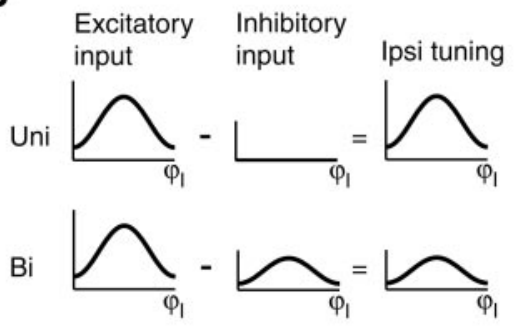

d

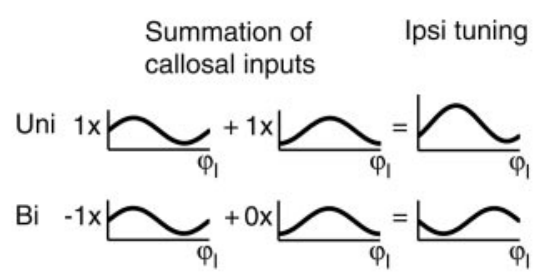

e

Left motor Right motor cortex Control signal cortex

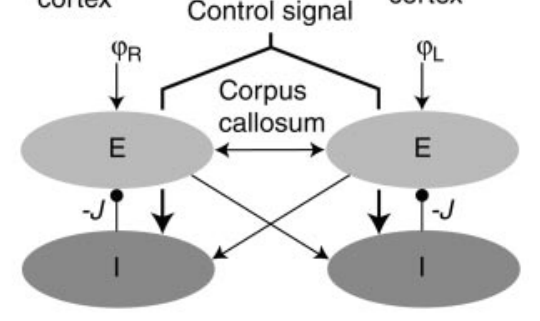

$\longleftarrow$ Excitation

Figure 8. The callosal inhibition mechanism. $a$, An example of an excitatory cell (light cell) that receives an instruction input that codes for the contralateral arm and a single callosal input that codes for the ipsilateral arm. The callosal input is conveyed by a direct excitatory pathway and via a local inhibitory interneuron (dark cell). The numbers indicate the connection weights. $b$, The excitatory callosal inputs, inhibitory callosal inputs, and total callosal inputs during unimanual movements (Uni) and during bimanual movements (Bi). $c$, An example of a cell that receives two callosal inputs. $d$, The callosal inputs from two sources and their sum during unimanual movements (Uni) and during bimanual movements (Bi).e, The callosal inhibition mechanism is incorporated into a model of two coupled networks, each including an excitatory and inhibitory population of cells.

homogeneous tuning properties over the population of cells. Models of this type, known as mean-field models, are inadequate to describe the heterogeneity in our system. We, in contrast, hypothesize that each excitatory cell receives callosal afferents from a small random subset of cells in the contralateral hemisphere. To account for the observed correlation between the ipsilateral and contralateral PDs we assume a preference for connecting pairs of cells with instruction inputs having similar PDs. As demonstrated above, having heterogeneous callosal connection strengths generates large shifts in the ipsilateral PDs. We, therefore, assume random independent callosal connection strengths, drawn from a uniform distribution between a minimum and maximum value. To illustrate the importance of this heterogeneity, we show in Figure $9 a$ that the mean shift of the ipsilateral PDs increases with the variability of the callosal connection strengths. In these simulations, the mean connection strength is fixed, and the width of the distribution is varied. The variability index ( $x$-axis) is defined as half the distribution width normalized by the mean. Thus, the point of lowest variability corresponds to homogeneous connection strengths, whereas the point of highest variability corresponds to the connection strengths being distributed between zero and twice the mean value.

The gain of the inhibitory interneurons $J$ plays an important role in determining the strength of the nonlinear effects. As $J$ increases, the contribution of the inhibitory callosal pathway to the response during bimanual movements is enhanced relative to the excitatory contribution. As a result, the ipsilateral PD shifts increase (Fig. $9 b$, solid line), and the correlation between $\theta_{b i}^{I}$ and $\theta_{u n i}^{C}$ decreases (Fig. $9 b$, dashed line). Here, we define the correlation between the two PDs as the mean of $\cos \left(\theta_{b i}^{I}-\theta_{u n i}^{C}\right)$, such that a correlation of 1 implies that the PDs are identical and a correlation of -1 implies that the PDs are exactly opposite. Notice that $\theta_{b i}^{I}$ and $\theta_{u n i}^{C}$ become anticorrelated when $J$ is increased beyond 1 . At this regime, the inhibition outweighs the excitation such that most tuned callosal inputs flip their PD. Additionally, for small $J$ the ipsilateral modulation amplitudes are suppressed as $J$ in- a

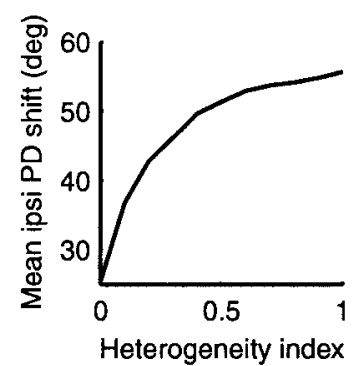

b

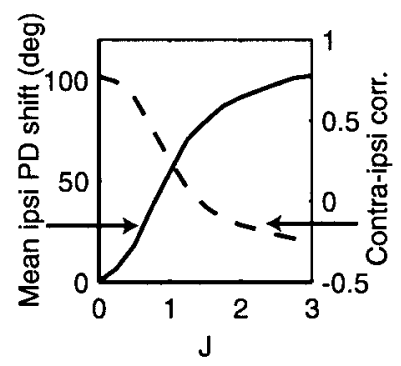

C

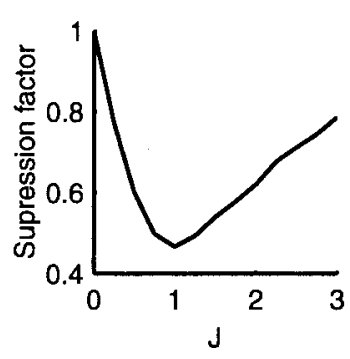

d

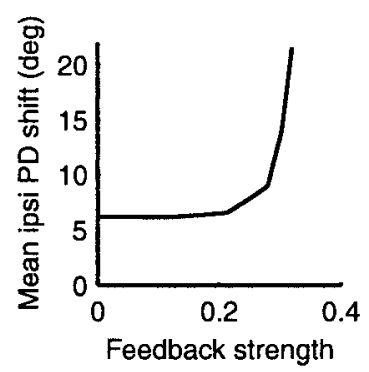

Figure 9. Behavior of the network model. $a$, The mean shift in the ipsilateral PD versus the heterogeneity index, which quantifies the width of the connection strength distribution. The heterogeneity index is defined as half the distribution width, normalized by the mean connection strength 0.25 . b, Gain $J$ of the inhibitory cells versus the mean shift of the ipsilateral PD (solid line) and the correlation between the ipsilateral and contralateral representations (dashed line). This correlation is defined as the mean of $\cos \left(\theta_{b i}^{\prime}-\theta_{u n i}^{c}\right)$. c, The suppression factor of the ipsilateral amplitudes versus $J$. The suppression factor is defined as the ratio between the mean of $a_{b i}^{l}$ and the mean of $a_{u n i}^{l} d$, Mean ipsi PD shift versus the intrahemispherical feedback strength $w$ for $J=0.2$.

creases (Fig. 9c). Here, we define the suppression factor ( $x$-axis) as the ratio of the mean of $a_{b i}^{I}$ and the mean of $a_{u n i}^{l}$. Notice that as $J$ is increased beyond 1 the inhibition dominates the bimanual responses, and large ipsilateral amplitudes are generated. We 
conclude that in the intermediate regime of $J$ around 1 the inhibition is most effective in suppressing the ipsilateral representation and removing its correlation with the contralateral representation. We, therefore, chose a value of $J=1$ in our simulations.

In the simulations described so far, we excluded intrahemispherical cortical interactions, so that the tuning of a cortical cell is generated entirely by its long-range afferents. However, it is believed that intrahemispherical interactions play an important role in sharpening the tuning of cortical neurons. We, therefore, added recurrent connections to the model and tested how this affects the modification of the ipsilateral representation. We assumed that each excitatory neuron receives inputs from a small number of randomly chosen neurons within the same network. In this case, the ipsilateral tuning during bimanual movements of a given neuron is modified because of two factors: (1) the local inhibition; and (2) the modification of the ipsilateral tuning of its presynaptic neurons within the same hemisphere. The latter factor provides an intra hemispherical feedback loop that amplifies the nonlinear effect. Thus, even if the inhibitory gain is small, a substantial modification of the ipsilateral PDs can be obtained by adding recurrent connections (Fig. 9d). For simplicity, we omitted the intrahemispheric connections in the simulations below.

Our network model reproduces the main effects we observed in the tuning properties of the cells. The architecture we assumed for the callosal connections indeed generates unimanual contralateral and ipsilateral PDs that are different, yet correlated (Fig. $2 a$, solid line). The inhibitory mechanism we assumed in the model produces bimanual offsets that are substantially smaller than those predicted by the linear hypothesis (Fig. $4 c, d$ ). Additionally, the bimanual offsets are similar to the contralateral offsets, as shown by their similar means and SDs and their high correlation coefficient (0.88). The ipsilateral tuning of the model cells exhibits modifications similar to those observed in the data. The amplitudes of the ipsilateral tuning show a trend of suppression (Fig. $5 b$, dots in right scatter plot), whereas the ipsilateral PDs are shifted substantially (Fig. $5 a$, right solid line). In contrast to the single-cell model prediction, these ipsilateral PD shifts are independent of the difference between the unimanual PDs (Fig. $7 b$, dots), because the inhibition that causes these shifts is unrelated to the contralateral PD. Furthermore, the correlation between the modified ipsilateral PDs and the contralateral PDs is very small (Fig. $2 b$, solid line). Finally, the modification of the contralateral representation is much smaller than that of the ipsilateral representation (Fig. 5, left). This effect stems from the fact that the contralateral tuning is not directly modified by the callosal inhibition and is only affected by the interhemispheric feedback.

Although our model reproduces the main effects that we observed in the data, there is a systematic tendency of the model to produce PD differences that are smaller than the experimental PD differences (Figs. 2, 5a, 7b). However, it is important to note that the experimentally extracted PDs have a noisy component that is attributable to the limited number of trials for each movement. Indeed, when we add noise to the simulated tuning curves that is comparable with the experimental noise, this discrepancy is largely removed (data not shown). Additionally, the model generates a variability in the offsets that is smaller than the observed variability (Fig. 4). This may be a consequence of the particular choice of neuronal heterogeneity in our model, namely a uniform distribution of neuronal gains.

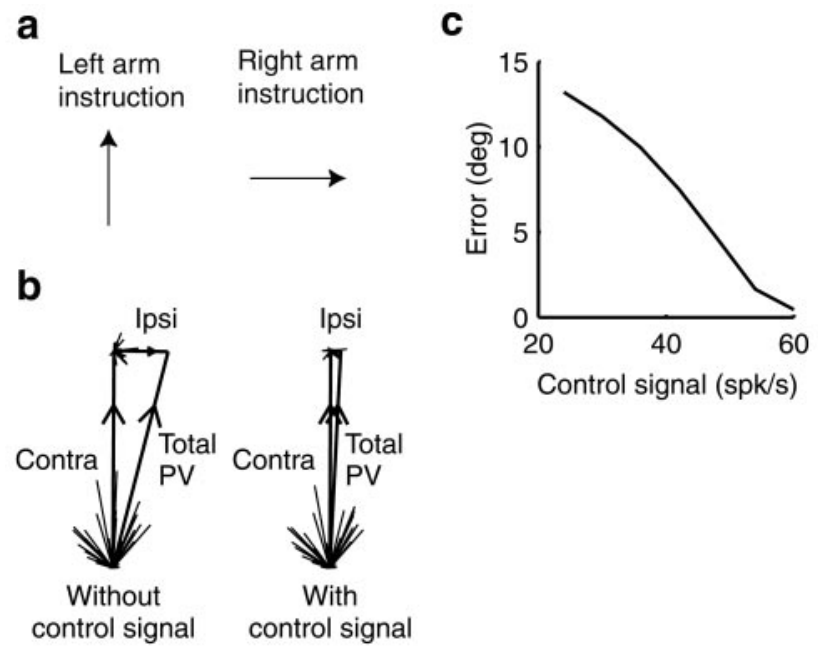

Figure 10. The modification of the arm representation during bimanual movements implies bimanual decoupling. $a$, We consider a bimanual movement of the left hand upward and the right hand rightward. $b$, The bimanual coupling in the absence of the control signal (left) and in the presence of the control signal (right). The population vectors in both cases (total PV) are composed of a contribution of the contralateral components of the bimanual responses (vertical vectors) and a contribution of the ipsilateral components (horizontal vectors). The lines represent the populations of vectors. c, The error in the direction of movement can be continuously modulated by the control signal.

\section{Implications for bimanual coupling}

What does the nonlinear cortical representation of bimanual movements imply for movement control? Because of the substantial heterogeneity in the experimental data, it is difficult to address this issue using the small samples of recorded cells directly. However, we can study the readout of the system by sampling a large number of cells from our network model. We use the concept of population vector (PV), which states that the hand velocity vector is computed from the cell activities $r_{i}$ by:

$$
\overrightarrow{\mathrm{PV}}=\sum_{\mathrm{i}} r_{i} \vec{e}_{i}
$$

where $\vec{e}_{\mathrm{i}}$ are unit vectors directed at the cell PDs (Georgopoulos et al., 1988). The PV has been shown to be a good predictor of the instantaneous contralateral hand direction during unimanual movements (Moran and Schwartz, 1999). Here, we assume that during bimanual movements the PV of each hemispheric population, computed by $\theta_{u n i}^{C}$ serves as a predictor of the movement direction of the contralateral arm. To see the effect of the bimanual representation on the prediction of the PV, consider a task in which a subject is required to move the left hand upward and the right hand rightward (Fig. 10a). First, we compute the right hemisphere PV in the absence of the control signal. In this case, the inhibitory interneurons are mostly subthreshold, such that the ipsilateral representation is scarcely modified. The PV (Fig. $10 b$, vector designated total PV) is composed of two vectors corresponding to the contralateral (vertical vector) and ipsilateral (horizontal vector) components of the bimanual responses. Ideally, the PV should point upward, but the ipsilateral component exerts an unintentional rightward velocity. Notice that the ipsilateral component results from the correlation between the contralateral and ipsilateral PDs. This phenomenon of coupling between the directions of movement of the two arms, termed spatial bimanual coupling, is well known in human psychophysics (Franz, 1997; Heuer et al., 1998) and has been attributed to neural 
cross-talk between the hemispheres (Cattaert et al., 1999; Cardoso de Oliveira et al., 2001).

When the control signal is turned on, the ipsilateral representation is suppressed and modified in its PD such that it loses its correlation with the contralateral PD. As a result, the bias in the $\mathrm{PV}$ is considerably reduced, and the arms are effectively decoupled (Fig. 10b, right). This decoupling is consistent with recent local field potential measurements that indicate that the correlation between the electrical activities of the two motor cortices is suppressed during the execution of the same type of bimanual movements as in our experiments (Cardoso de Oliveira et al., 2001). Thus, our model shows that a control signal that recruits local inhibitory interneurons can suppress the neural cross-talk between the hemispheres. Interestingly, continuously increasing the control signal modulates the degree of neural cross-talk continuously, between the high coupling scenario depicted on the left of Figure $10 b$ and the low coupling scenario depicted on the right. Figure $10 c$ shows how the error in the direction of movement that is induced by the neural cross-talk gradually decreases with the increase in the control signal. This gradual change occurs because more interneurons are activated, and, thus, more neurons undergo a modification of their ipsilateral representations.

\section{Discussion}

We have shown that during a bimanual visually guided reaching task to two different targets, the cortical representation of the ipsilateral arm movement is suppressed relative to the representation of the same arm when it is moved alone. Two effects combine to generate this suppression: (1) the modulation amplitudes of the directional tuning of the cell to the ipsilateral arm tend to be smaller in the bimanual condition; and (2) the PDs of the ipsilateral tuning are randomly shifted such that they lose the correlation with the contralateral PDs that is observed in the unimanual condition. These effects help demonstrate why the bimanual responses differ from the sum of the responses of the two constituent unimanual movements. We have explained these bimanual responses with a model composed of two coupled hemispheric populations, in which the callosal projections generate the cortical representation of the ipsilateral arm. Interestingly, the interhemispheric coupling tends to move the two arms in directions that are similar in extrinsic space rather than in directions that involve activation of homologous muscles, as would be expected if the activity of motor cortical cells simply reflects muscle activations. We have shown that recruitment of inhibitory interneurons during bimanual movements by an external control signal can account for the observed changes in the ipsilateral representation. This mechanism relies on two elements to suppress the tuning modulation depths and randomly shift the PDs: (1) threshold nonlinearity of the local inhibitory interneurons; and (2) heterogeneity of the callosal connection strengths. We have shown that the proposed mechanism of callosal inhibition enables a flexible context-dependent modulation of the degree of bimanual coupling.

Our conclusion that the interhemispheric cortical influences undergo suppression during bimanual reaching movements seems counter-intuitive and calls for an explanation. We suggest that the "default mode" of the motor system is a mode of strong interhemispheric coupling that helps coordinate the arms in bimanual tasks that require fine control of the relative motion of the two arms. Indeed, daily experience and behavioral studies show that limbs tend to coordinate automatically and that tasks that deviate from this natural coordination are difficult (Kelso et al., 1979; Franz et al., 1991; Weisendanger et al., 1996). Addition- ally, evidence for the importance of the corpus callosum for bimanual coordination comes from observations that normal subjects perform better than callosal subjects on certain bimanual tasks (Preilowsky, 1972; Serrien et al., 2001; Kennerley et al., 2002). However, in many bimanual movements, the precise coordination of the relative movements of the two arms is not required, and some degree of decoupling is advantageous. In our particular bimanual task, which requires visually guided movements to two different targets, an accurate execution of the movement of the two arms independently is more important for successful task performance. Thus, the monkey may adopt a strategy of suppressing the natural tendency of the arms to couple. This is corroborated by evidence that callosal patients perform better in tasks that require two simultaneous different movements of the two arms (Franz et al., 1996). The ipsilateral-related responses during unimanual movements might reflect bilateral coupling that exists by default in the motor cortex. Alternatively, these signals might help maintain the unmoved arm in place, by counterbalancing signals arriving through lower-level bilateral coupling (e.g., at the spinal cord).

\section{Other studies of bilateral responses}

Here, we introduced a novel model for the cortical representation of bimanual movements (Eq. 9) that generalizes the common cosine tuning model of the unimanual movement representation. The advantage of this model is that it allows us to disassociate the contribution of the contralateral and ipsilateral movements to the bimanual response and to compare these components with the corresponding unimanual representations. It would be interesting to further test this model in future experiments with a more general repertoire of directions and amplitudes of movements.

Our analysis was based on pooling the cells from SMA, PM, and MI. Inspection of the data from each area separately did not yield substantial differences in either the ipsilateral or the bimanual responses. A recent study (Cisek et al., 2003) found a strong correlation between the contralateral and ipsilateral PDs in PM, similar to the correlations reported here for the entire population of SMA PM and MI cells, but much weaker correlations in caudal MI. This discrepancy probably stems from the fact that our MI recordings were located more rostrally. Indeed, the abovementioned study has found evidence for a systematic decrease in these correlations as one progresses from rostral to caudal directions. Furthermore, other studies (Crammond and Kalaska, 1996, 2000) also show that movement tuning properties change along the rostrocaudal axis. Our study has found an overall tendency of suppression of bimanual movements relative to the sum of the unimanual responses. Other studies of responses of cortical cells to bimanual movements have reported examples of enhancements as well as suppressions (Tanji et al., 1987, 1988; Kermadi et al., 2000; Donchin et al., 2002). It is important to stress that the presence of bimanual enhancements in part of the cell population is consistent with our model, for the following reasons. First, the random shifting of the PDs may increase the response to certain configurations of bimanual movements even when the modulation amplitude is suppressed. Additionally, the broad distribution of tuning properties across the population implies that many cells undergo an enhancement of tuning modulation depths. Finally, the model includes a population of inhibitory cells that only respond during bimanual movements.

\section{Additional evidence for callosal inhibition}

Experimental evidence from other studies supports our hypothesis that callosal afferents to the motor cortex can induce local inhibition. 
Electrical stimulation in an anesthetized cat showed the existence of excitatory and inhibitory callosal projections (Asanuma and Okuda, 1962). Transcranial magnetic stimulation (TMS) studies in humans have shown that a conditioning stimulus on one motor cortex suppresses the response of the opposite motor cortex (Ferbert et al., 1992; Lazzaro et al., 1999) to a local testing stimulus, an effect that is absent in callosal patients (Meyer et al., 1995; Schnitzler et al., 1996). In the framework of our model, the suppression of the response to the testing stimulus is attributed to the inhibition by the conditioning stimulus. In other TMS experiments, in which the conditioning and testing stimuli were much weaker, a facilitatory effect was observed (Ugawa et al., 1993). In this case, we claim that the interneurons were subthreshold.

Nonlinear suppressive effects on the activity of a local group of neurons by the activations of a remote population have also been observed in the primary visual cortex (Kapadia et al., 1995; Toth et al., 1996; Polat et al., 1998). Analogous to our interpretation, it was suggested that this nonlinearity is attributable to the recruitment of local inhibitory interneurons (Stemmler et al., 1995; Sommers et al., 1996) by cortical long-range connections. These observations suggest that the modulation of cortical processing via local inhibitory circuitry is a general principle of the cortical function.

\section{References}

Asanuma H, Okuda O (1962) Effects of transcallosal volleys on pyramidal tract cell activity of cat. J Neurophysiol 25:198-208.

Ben-Yishai R, Bar-Or RL, Sompolinsky H (1995) Theory of orientation tuning in visual cortex. Proc Natl Acad Sci USA 92:3844-3848.

Cardoso de Oliveira S, Gribova A, Donchin O, Bergman H, Vaadia E (2001) Neural interaction between motor cortical hemispheres during bimanual and unimanual arm movements. Eur J Neurosci 14:1881-1896.

Cattaert D, Semjen A, Summers JJ (1999) Simulating neural cross-talk model of between hand interference during bimanual circle drawing. Biol Cybern 81:343-358.

Cisek P, Crammond DJ, Kalaska JF (2003) Neural activity in primary motor and dorsal premotor cortex in reaching tasks with the contralateral versus ipsilateral arm. J Neurophysiol 89:922-942.

Crammond DJ, Kalaska JF (1996) Differential relation of discharge in primary motor cortex and premotor cortex to movements versus actively maintained postures during a reaching task. Exp Brain Res 108:45-61.

Crammond DJ, Kalaska JF (2000) Prior information in motor and premotor cortex: activity during the delay period and effect on pre-movement activity. J Neurophysiol 84:986-1005.

Donchin O, Gribova A, Steinberg O, Bergman H, Vaadia E (1998) Primary motor cortex is involved in bimanual coordination. Nature 395:274-278.

Donchin O, Gribova A, Steinberg O, Mitz AR, Bergman H, Vaadia E (2002) Single-unit activity related to bimanual arm movements in the primary and supplementary motor cortices. J Neurophysiol 88:3498-3517.

Ferbert A, Priori A, Rothwell JC, Day BL, Colebatch JG, Marsden CD (1992) Interhemispheric inhibition of the human motor cortex. J Physiol (Lond) 453:525- 546.

Franz EA (1997) Spatial coupling in the coordination of complex actions. Q J Exp Psychol A 50:684-704.

Franz EA, Zelaznik HN, McCabe G (1991) Spatial topological constraints in a bimanual task. Acta Psychol 77:137-151.

Franz EA, Eliassen JC, Ivry RB, Gazzaniga MS (1996) Dissociation of spatial and temporal coupling in the bimanual movements of callosotomy patients. Psychol Sci 7:306-310.

Georgopoulos AP, Kalska JF, Caminiti R, Massey JT (1982) On the relations between the direction of two dimensional arm movements and cell discharge in primate motor cortex. J Neurosci 2:1527-1537.

Georgopoulos AP, Kettner RE, Schwartz AB (1988) Primate motor cortex and free arm movements to visual targets in three-dimensional space. II.
Coding of the direction of movement by a neuronal population. J Neurosci 8:2928-2937.

Heuer H, Spijkers W, Kliensorge T, van der Loo H (1998) Period duration of physical and imaginary movement sequences affects contralateral amplitude modulation. Q J Exp Psychol A 51:755-779.

Kapadia MK, Ito M, Gilbert CD, Westheimer G (1995) Improvement of visual sensitivity by change in local context: parallel studies in human observers and in V1 of alert monkeys. Neuron 15:843-856.

Kelso JAS, Southard DL, Goodman D (1979) On the coordination of twohanded movements. Psychol Hum Percept Perform 5:229-238.

Kennerley SW, Diedrichsen J, Hazeltine E, Semjen A, Ivry RB (2002) Callosotomy patients exhibit temporal uncoupling during continuous bimanual movements. Nat Neurosci 5:376-381.

Kermadi I, Liu Y, Rouiller EM (2000) Do bimanual motor actions involve the dorsal premotor (PMd), cingulate (CMA) and posterior parietal (PPC) cortices? Comparison with primary and supplementary motor cortical areas. Somatosens Mot Res 17:255-271.

Lazzaro VD, Oliviero A, Profice P, Insola A, Mazzone P, Tonali P, Rothwell JC (1999) Direct demonstration of interhemispheric inhibition of the human motor cortex produced by transcranial magnetic stimulation. Exp Brain Res 124:520-524.

Lecas JC, Requin J, Anger C, Vitton N (1986) Changes in neuronal activities of the monkey precentral cortex during preparation for movement. J Neurophysiol 56:1680-1702.

Meyer BU, Roricht S, Grafin von Einsiedel HG, Kruggel F, Weindl A (1995) Inhibitory and excitatory interhemispheric transfers between motor cortical areas in normal humans and patients with abnormalities of the corpus callosum. Brain 118:429-440.

Moran DW, Schwartz AB (1999) Motor cortical representation of speed and direction during reaching. J Neurophysiol 82:2676-2692.

Polat U, Mizobe K, Pettet MW, Kasamutsu T, Norcia AM (1998) Collinear stimuli regulate visual responses depending on cell's contrast threshold. Nature 391:580-584.

Preilowsky BFB (1972) Possible contribution of the anterior forebrain comissures to bilateral motor coordination. Neuropsychologia 10:267-277.

Schnitzler A, Kessler KR, Benecke R (1996) Transcallosaly mediated inhibition of interneurons within human primary motor cortex. Exp Brain Res 112:381-391.

Serrien DJ, Nirkko AC, Wiesendanger M (2001) Role of the corpus callosum in bimanual coordination: a comparison of patients with congenital and acquired callosal damage. Eur J Neurosci 14:1897-1905.

Sommers DC, Toth LJ, Todorov E (1996) Variable gain control in local cortical circuitry supports context-dependent modulation by long-range connections. In: Lateral interactions in the cortex: structure and function (Sirosh J, Mikkulainen R, eds). Austin, TX: The UTCS Neural Networks Research Group, University of Texas.

Steinberg O, Donchin O, Gribova A, Cardoso de Oliveira S, Bergman H, Vaadia E (2002) Neuronal populations in primary motor cortex encode bimanual arm movements. Eur J Neurosci, 15:1371-1380.

Stemmler M, Usher M, Neibur E (1995) Lateral interactions in primary visual cortex: a model bridging physiology and psychology. Science 269:1877-1880.

Swinnen SP (2002) Intermanual coordination: from behavioral principles to neural-network interactions. Nat Rev Neurosci 3:350-361.

Tanji J, Kazuhiko O, Sato KC (1987) Relation of neurons in the nonprimary motor cortex to bilateral hand movement. Nature 327:618-620.

Tanji J, Kazuhiko O, Sato KC (1988) Neural activity in cortical motor areas related to ipsilateral, contralateral, and bilateral digit movements of the monkey. J Neurophysiol 60:325-343.

Toth LJ, Rao SC, Kim DS, Somers D, Sur M (1996) Subthreshold facilitation and suppression in primary visual cortex revealed by intrinsic signal imaging. Proc Natl Acad Sci USA 93:9869-9874.

Ugawa Y, Hanajima R, Kanazawa I (1993) Interhemispheric facilitation of the hand area of the human motor cortex. Neurosci Lett 160:153-155.

Weisendanger M, Rouiller EM, Kazennikov O, Perrig S (1996) Is the supplementary motor area a bilaterally organized system? Adv Neurol 70:85-93. 